\title{
Limitações das Atuais Soluções em Software: Uma Visão para Desenvolvimento de Ferramentas em Gestão de Projetos
}

\author{
Dani Juliano Czelusniak ${ }^{1}$, Alessandro de Oliveira Binhara ${ }^{2}$, Carlos Eduardo Yamasaki Sato $^{3}$, \\ Dario Eduardo do Amaral Dergint ${ }^{4}$, Luiz Alberto Pilatti ${ }^{5}$ \\ ${ }^{1}$ Programa de Pós Graduação em Engenharia de Produção, Centro Federal de Educação \\ Tecnológica do Paraná, Av. Monteiro Lobato s/n, KM 4, Ponta Grossa, PR, Brasil \\ dani.juliano@gmail.com \\ ${ }^{2}$ Programa de Pós Graduação em Tecnologia, Centro Federal de Educação Tecnológica do \\ Paraná, Av. Sete de Setembro, 3165, Bairro Rebouças, Curitiba, PR, Brasil \\ binhara@gmail.com
}
${ }^{3}$ Science and Technology Policy Research (SPRU), University of Sussex, Freeman Centre, Falmer, Brighton, East Sussex, BN1 9QE, United Kingdom c.e.y.sato@sussex.ac.uk

${ }^{4}$ Programa de Pós Graduação em Tecnologia, Centro Federal de Educação Tecnológica do Paraná, Av. Sete de Setembro, 3165, Bairro Rebouças, Curitiba, PR, Brasil dergint@ppgte.cefetpr.br
${ }^{5}$ Programa de Pós Graduação em Engenharia de Produção, Centro Federal de Educação Tecnológica do Paraná, Av. Monteiro Lobato s/n, KM 4, Ponta Grossa, PR, Brasil
luiz.pilatti@terra.com.br

\begin{abstract}
Resumo - Diante do atual estado da evolução tecnológica, a Gestão de Projetos torna-se cada vez mais importante dada a crescente complexidade dos projetos. Neste cenário as soluções de software existentes no mercado mostram-se ineficientes face ao dinamismo e aumento do número de variáveis que precisam ser controladas pelo gestor. Este artigo apresenta um estudo que visa verificar as atuais limitações dos softwares de auxílio ao gestor de projetos, demonstrando como novas tecnologias de desenvolvimento de software podem contribuir para a construção de ferramentas mais eficazes. Sistemas de informações construídos sobre os novos modelos de desenvolvimento podem ajudar no controle de projetos onde as atuais ferramentas apresentam limitações. A metodologia utilizada neste estudo foi a revisão bibliográfica para esclarecer quais são os novos modelos de desenvolvimento, como desenvolve-se um projeto e como aplicam-se suas ferramentas de controle. Este trabalho termina apresentando sugestões em que o uso destas novas metodologias demonstra bom potencial para futuras implementações pelo fato de serem flexíveis, por oferecer respostas em um menor prazo e por oferecer formas de tratar a não linearidade característica dos novos projetos.
\end{abstract}

Palavras-chave: gestão de projetos, sistema multilinguagem, sistemas multiplataforma.

Abstract - In the current state of the technological evolution, the Project Management becomes more important with the increasing complexity in the projects. In this scenario, the software solutions become inefficient, considering the dynamism and the increasing number of variables which need to be controlled by the project manager. This article shows a study that aims to verify the current limitations of softwares which support the project manager, demonstrating how the new software development technologies can contribute for the construction of more efficient development tools. Information systems, built with new development models, can help in project control, where the present tools have limitations. The methodology used in this study was the literature review to show which are the new development models, how a project is developed and where its 
control tools are applied. This work concludes presenting suggestions where the use of these new methodologies demonstrates good potential for future implementations due to the fact that they are flexible, by offering answers quickly and by offering ways to deal with the non linearity which characterizes new projects.

Key-words: project management, multilanguage systems, multiplataform systems.

\section{Introdução}

É cada vez mais comum em ambientes corporativos a aplicação de metodologias orientadas a projetos para o desenvolvimento e produção de bens tangíveis e serviços. Com o advento da modernização, sua gestão torna-se cada vez mais complexa implicando em custos cada vez maiores em decorrência de vários fatores como a evolução natural dos processos produtivos, equipe multidisciplinar envolvida, extenso número de variáveis a serem gerenciadas, dentre outros.

Em se tratando de software, seu projeto é uma atividade empreendida para obtenção de um sistema segundo requisitos que atendam condições pré-estabelecidas pelo cliente, o utilizador do software. Este processo poderá estender-se com o aumento das variáveis envolvidas, fazendo com que sua administração torne-se confusa caso não existam técnicas e ferramentas de gerenciamento que possam garantir um delineamento bem sucedido ao projeto [1].

Atualmente muitos desses projetos são controlados utilizando ferramentas de software e metodologias pré-concebidas, como por exemplo o modelo RUP (Rational Unified Process) utilizado para gestão de projetos de desenvolvimento de software. Este prevê uma metodologia aliada a um conjunto de ferramentas de software. A grande problemática do uso destas metodologias é que na maioria dos casos elas não se adaptam à empresa, o que culmina em problemas nos processos produtivos, estes por sua vez acabam sendo obrigados a adaptar-se à ferramenta.

Para tratar este problema, apresenta-se na seqüência uma breve introdução sobre gestão de projetos e o uso de ferramentas de software em sua gestão. Logo após, aborda-se a dificuldade do uso de ferramentas tradicionais pela impossibilidade da adequação e customização das mesmas para as necessidades de cada projeto. Este artigo encerra apresentando as vantagens potenciais do uso de novas técnicas de programação para a construção de ferramentas multiplataforma que auxiliem na gestão de projetos de alta complexidade.

\section{Projeto e sua gestão}

Toda a atividade humana caracteriza-se pela definição de objetivos a serem atingidos tendo prazos e determinadas condições a serem seguidas. Neste sentido, qualquer atividade humana pode ser considerada um projeto. Um projeto é um empreendimento temporário que possui início, desenvolvimento e encerramento bem definidos com objetivo de fornecer ao seu término um produto ou serviço, dentro de um prazo de tempo previamente estipulado obedecendo a um orçamento [2].

Com o objetivo de criar e manter uma padronização dos principais conceitos e técnicas utilizadas na gestão de projetos, em 1969 nos EUA foi fundado o Project Management Institute (PMI), que é o instituto que norteia o tema Gestão de Projetos. O PMBOK (Project Management Body of Knowledge) é fruto dos esforços da PMI sendo considerado o guia do conhecimento denominado na gestão de projetos. Segundo a PMI existem mais de 950 mil cópias em circulação no mundo.

Neste contexto, o PMBOK possui nove capítulos conforme identificados abaixo, cada um contendo uma área de concentração onde pode-se visualizar os componentes principais da gestão de projetos [3]. 


\begin{tabular}{|c|c|c|}
\hline Cap. & $\begin{array}{l}\text { Foco da } \\
\text { Gestão }\end{array}$ & Breve descritivo \\
\hline 4 & Integração & $\begin{array}{l}\text { Planejamento } \\
\text { projeto: } \\
\text { planejamento não é um } \\
\text { processo que ocorre } \\
\text { apenas em um } \\
\text { momento específico } \\
\text { ante à sua execução, } \\
\text { mas contínuo e corre } \\
\text { em paralelo com o } \\
\text { processo de execucão. }\end{array}$ \\
\hline 5 & Escopo & $\begin{array}{l}\text { Resultado final do } \\
\text { processo, o produto ou } \\
\text { serviço; divide-se em } \\
\text { planejamento } \\
\text { definição. } \\
\text { Planejamento: } \\
\text { descrição concisa dos } \\
\text { produtos ou serviços } \\
\text { que serão fornecidos; } \\
\text { Definição: descrição } \\
\text { detalhada dos produtos } \\
\text { ou serviços que serão } \\
\text { fornecidos; }\end{array}$ \\
\hline 6 & Tempo & $\begin{array}{l}\text { Realização das } \\
\text { atividades necessárias } \\
\text { para a realização do } \\
\text { produto ou serviço } \\
\text { (cronograma) }\end{array}$ \\
\hline 7 & Custos & $\begin{array}{l}\text { Planejamento de } \\
\text { recursos orçamentários } \\
\text { do projeto }\end{array}$ \\
\hline 8 & Qualidade & $\begin{array}{l}\text { Controle de qualidade } \\
\text { do produto ou serviço } \\
\text { do projeto. Engloba os } \\
\text { processos de controle } \\
\text { do resultado final do } \\
\text { projeto e também do } \\
\text { processo } \\
\text { administração } \\
\text { projeto; }\end{array}$ \\
\hline 9 & $\begin{array}{l}\text { Recursos } \\
\text { Humanos }\end{array}$ & $\begin{array}{l}\text { Organização da equipe } \\
\text { de projeto }\end{array}$ \\
\hline 10 & $\begin{array}{l}\text { Comunica- } \\
\text { ções }\end{array}$ & $\begin{array}{l}\text { Trâmites } \\
\text { administrativos do } \\
\text { projeto }\end{array}$ \\
\hline 11 & $\begin{array}{l}\text { Eventualida- } \\
\text { des (riscos) }\end{array}$ & $\begin{array}{l}\text { Eventos externos } \\
\text { afetam o curso } \\
\text { projeto }\end{array}$ \\
\hline 12 & Suprimentos & $\begin{array}{l}\text { Aquisição de insumos } \\
\text { necessários ao projeto }\end{array}$ \\
\hline
\end{tabular}

Quadro 01 - Componentes da gestão de projetos segundo o PMBOK
A necessidade de novos mercados e a busca por um crescimento contínuo das empresas tornaram a gestão de projetos requisito básico para a sobrevivência dentro da comunidade global sendo este uma premissa para a sobrevivência e garantia de crescimento.

Preparar uma empresa para atingir o meio internacional, está sendo uma necessidade cada vez maior não só de grandes, mas também de pequenas e médias empresas que possuem objetivos específicos [4].

No atual estado de evolução, a tecnologia da informação e comunicação tem acelerado tanto a geração quanto 0 consumo de produtos tecnológicos, ou seja, as empresas com o objetivo de se manterem competitivas precisam manter-se atualizadas para garantir seu crescimento consumindo portanto, bens tecnológicos.

A empresa, de posse da tecnologia adequada, tenta fazer com que a informação flua de forma rápida e consistente para que os objetivos desejados sejam atingidos com sucesso e com a qualidade necessária [5]. A necessidade de sistemas de informação inteligentes, flexíveis e adaptáveis que possibilitem uma agilidade na busca e tratamento da informação, tornou-se vital para a gestão de projetos dentro das organizações.

\section{Empresas e projetos: situação atual}

É nebulosa a forma como a gestão de projetos é executada dentro do contexto organizacional, sejam eles projetos de melhorias nos processos, produtos diferenciados para clientes específicos, novos produtos a serem lançados no mercado, entre outros. Este assunto é altamente relevante pelo fato de que cada empresa, em determinado instante, cria ou adapta uma metodologia para atender a uma determinada situação, fazendo com que as ferramentas de software existentes no mercado não atendam suas necessidades. A necessidade de adaptação da empresa ou parte dela à ferramenta, atropelando processos industriais e normas internas procedimentadas, pode gerar problemas administrativos e atrasos em sua linha de produção, independente da indústria produzir serviços ou bens tangíveis e intangíveis. Quando estes problemas não são adequadamente gerenciados, a qualidade do produto final pode ser comprometida, a expectativa do cliente pode não ser atendida, e a equipe que precisa conviver com ansiedades e conflitos durante o ciclo de vida do projeto pode ter sua produtividade reduzida [6]. 


\section{Ferramentas de software na gestão}

Os avanços tecnológicos que permeiam a humanidade, aliados ao barateamento de ferramentas informatizadas, acabaram por facilitar o acesso de todos à tecnologia em questão. Computadores vêm sendo utilizados em larga escala no auxílio à gestão, fazendo com que o uso de ferramentas de software torne-se "peça-chave", tanto para reduzir a possibilidade de erros como para aumentar o tempo de resposta do gestor, fazendo com que determinadas situações tornemse previsíveis com antecedência.

Vemos cada vez mais um crescimento nas ofertas de sistemas capazes de gerenciar projetos tanto de pequenos como de grande fabricantes de softwares. Os preços e recursos dos softwares também variam muito, encontrando inclusive sistema gratuitos e sistemas baseados em software livre (com o código aberto) que muitas vezes são clones de ferramentas proprietárias.

Comumente as arquiteturas destes softwares são predefinidas pelos seus fabricantes que empacotam estes sistemas como uma espécie de "caixa-preta" (com exceção dos softwares livres que possuem seu código aberto), a qual tenta suprir todas as necessidades do cliente e muitas vezes também já prevê uma metodologia a ser utilizada com processos e rotinas bem definidas. Isto não só limita o usuário que pretende realizar a gestão de seus projetos como também impõem novas rotinas, as quais não eram práticas dentro da organização. A empresa obriga-se a adequar este procedimento previsto inserindo novas práticas e alterando outras.

O RUP (metodologia utilizada para a gestão de projetos de software), por exemplo, estabelece quatro fases bem definidas no processo de gestão do projeto: concepção, elaboração, implementação e testes. Qualquer mudança que seja implementada no processo de gestão não é suportada pela ferramenta, isto "engessa" o processo e em um caso específico de mudança e recai sobre o gerente a responsabilidade, pois a ferramenta computacional não possui a flexibilidade necessária.

Devido às ferramentas serem construídas baseadas em processos previamente definidos sem a participação do usuário que irá fazer uso do sistema, existem poucas integrações com as ferramentas e bases de conhecimentos da empresa. Em se tratando de ferramentas de comunicação, atualmente uma das mais usadas é o e-mail. O e-mail não só é usado para agendar reuniões como para gerar especificações de requisitos, solução de dúvidas, documentação de etapas ou até mesmo como documento para provar inconsistências de comunicação que levam a gerar problemas nos resultados de projetos.

A não utilização do conhecimento gerado pela troca de e-mail e pelo uso de outras ferramentas entre os integrantes de uma equipe de projetos, leva à necessidade de alimentar manualmente as ferramentas de gestão de projeto. Por exemplo, uma alteração de prazo, que foi discutida dentro de um e-mail, tem que ser alterada e registrada numa ferramenta que faz a gestão do tempo do projeto e das atividades. Isto demanda tempo do gestor e encarece o processo como um todo.

A falta de integração entre as ferramentas em conjunto com a complexidade do fluxo de informações, culminam em que manter as informações atualizadas dentro das ferramentas de gestão torne-se tão caro quanto executar o projeto. O problema torna-se mais grave ainda quando não se consegue exigir que as pessoas atualizem os dados, por exemplo em projetos distribuídos, onde a informação não está em um só local ou gerenciada por apenas uma pessoa.

\section{Novas formas para implementação de ferramentas}

A situação ideal é que a ferramenta consiga adaptar-se ao funcionamento da empresa, fazendo com que seus processos internos possam ser mapeados e acompanhados como projetos, sem a necessidade de "reengenharias absurdas". Para isso, a ferramenta precisa ser modularizada, para que possa ser facilmente adaptada, cobrindo anseios dos gestores de projeto em curtos espaços de tempo; evolutiva para acompanhar o crescimento da empresa e o amadurecimento de seus produtos e serviços, garantindo-lhe os melhores resultados; e, não linear, para que o gestor consiga visualizar a empresa como um grande macro-processo.

Este macro-processo seria totalmente interdependente, podendo ser explodido em níveis de detalhamento mais e mais especializados, com granularidade suficiente para visualizar e isolar até mesmo pequenos pontos do processo, tornando a administração uma tarefa prazerosa onde o sistema torna-se quase um ser vivo capaz de prever e identificar problemas, sugerindo ações para correção com base nas decisões tomadas em outras situações.

\section{Uso do Software Livre}

Uma nova forma de desenvolvimento que vem obtendo muito sucesso na solução de problemas complexos por adotar um modelo 
distribuído é utilizada pela comunidade software livre. Software Livre é todo o software que tem seu código fonte aberto e em sua licença de uso concede a liberdade a seus usuários de copiar, distribuir e modificar seu código fonte. Este modelo tem como pano de fundo tecnológico o item inovação como chave do sucesso.

Como este modelo permite a total customização e adequação do software, mesmo soluções pequenas e pobres em recursos quando comparados a caras soluções proprietárias, conjugadas com outras soluções e adaptadas às necessidades específicas da empresa, podem se tornar ferramentas com ótimo custo benefício.

A disponibilidade do código fonte possibilita que ocorram integrações aos sistemas e bases de conhecimento, possibilitando uma interação que antes não era possível. Um exemplo interessante de integração e adaptação de softwares para criação de um completo ambiente de gerência de projetos de software é o Source Forge (www.sourceforge.net) que nada mais é que a integração de várias ferramentas livres em uma interface web. Nesta ferramenta podemos encontrar listas de discussão, fórum, site web, sistema de controle de versão de documentos, sistema de rastreamento de bugs, dentre outras. Um conjunto de inovações ocorreu sobre o então Source Forge e foi criado o GForge, que é uma nova versão do sistema com uma série de melhorias como sistemas de buscas, ranqueamento de projetos entre outras inovações.

Seguindo esta mesma visão de inovação em tecnologias, também estão sendo criados ambientes multiplataforma e multilinguagem, os quais permitem que uma aplicação que seja desenvolvida para a plataforma Microsoft $\AA$, por exemplo, seja capaz de funcionar dentro do ambiente Linux.

\section{Plataforma .Net e Mono}

Ao longo dos anos diversas linguagens de programação tem sido desenvolvidas para solucionar os mais variados problemas, utilizando as mais diversas abordagens. Mas 0 compartilhamento e a integração entre as linguagens são praticamente inexistentes ou de difícil implementação. Esta situação cria uma barreira para a integração entre diferentes ferramentas de gestão.

Hoje essa questão está sendo tratada pela nova plataforma de desenvolvimento da Microsoft, a .Net (leia-se dot net) que possibilita a integração transparente entre as linguagens de programação, o que poderá em um futuro próximo colaborar para melhorias na integração das ferramentas de gestão.
Apesar da plataforma .Net ser uma solução interessante ainda é uma ferramenta proprietária que possui seu código fonte fechado. Recentemente foi lançando da Internet um projeto chamado Mono, que é a implementação da plataforma .Net em software livre. Além de possibilitar rodar aplicações em diversas plataformas, pode-se utilizar múltiplas linguagens em um mesmo aplicativo. Isto representa a possibilidade de integração total entre sistemas e linguagens de programação.

Um dos grandes diferencias da plataforma Mono e o .Net é que por ser um software livre este possibilita que uma aplicação Mono seja embutida dentro de outra aplicação. Por exemplo, uma empresa que usa um sistema de agenda e correio eletrônico, uma ferramenta de gestão de cronograma, outra aplicação para gerencia de custos. Todas as aplicações em linguagens e plataformas distintas. Presumindo que todas as aplicações têm código fonte aberto, é possível desenvolver uma ferramenta de gestão de projeto que colete as informações em todas essas bases de conhecimento e as disponibilize-as em uma intranet, possibilitando a gerência de diversos projetos.

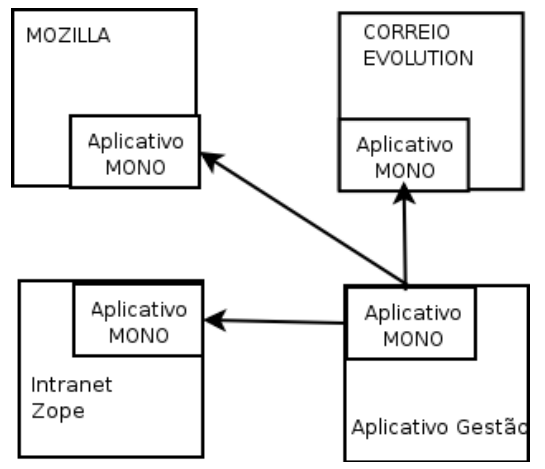

Figura 01 - Integração de software com o Mono

\section{Prevalência de objetos}

Outro conceito que deve auxiliar muito no desenvolvimento de ferramentas para gestão de projeto é a prevalência de objetos. Toda e qualquer ferramenta precisa armazenar os dados em disco rígido, e a maioria dos atuais sistemas de gestão usa sistema de banco de dados relacionais.

O grande problema do uso de sistemas de banco de dados é a sua baixa velocidade e a sua inflexibilidade na maneira de representar o conhecimento, pois neste modelo ele precisa ser representado no modelo de entidade $e$ relacionamentos. Atualmente a metodologia de programação orientada a objetos possibilita ao desenvolvedor de software grande flexibilidade ao 
poder representar o mundo computacional como objetos. Na maioria dos casos, a flexibilidade ganha com a utilização da orientação a objetos é prejudicada pela necessidade de se armazenar estes objetos em banco de dados relacionais.

Estes objetos poderiam ser armazenados em bancos de dados orientados a objetos, mas estes muitas vezes são muito caros e muito lentos se comparados ao banco de dados relacionais. O objetivo da prevalência de objetos é permitir que os objetos possam prevalecer na memória sem a necessidade de gravá-lo num banco de dados relacional ou orientado a objetos.

A primeira implementação de prevalência foi criada por Klaus Wuesfeld, criador do Prevayler (www.prevayler.org), uma camada de abstração de software escrita em Java que permite aos objetos permanecerem em memória, garantindo um aumento significativo de performance e a utilização de toda a flexibilidade da orientação à objetos. Usar e armazenar objetos em memória sem necessitar de uma estrutura estática de armazenamento de dados possibilita uma flexibilidade que antes não era possível nos sistemas de banco de dados relacionais.

Este conceito de prevalência de objetos já está disponível em várias linguagens diferentes como Ruby, Python, .Net e outras, e já é utilizado em grandes sistemas de gestão na Internet.

\section{Conclusões}

Muitas vezes as ferramentas de software oferecidas no mercado não possibilitam o tratamento de informações trocadas pelos componentes da equipe de projeto no decorrer de sua execução. Neste sentido, pode-se perceber que a capacidade de adaptação, reuso e potencial de inovação que estão inerentes nos softwares livres, conjugados com o uso de ferramentas de desenvolvimento que possibilitem a geração de aplicações que possam ser executadas na maioria dos sistemas operacionais, possibilitam a construção de ferramentas muito mais adaptadas às necessidades atuais da equipe de trabalho e do gestor de projetos.

Este modelo de desenvolvimento que se utiliza ferramentas que têm a capacidade de trabalhar de forma distribuída pode reduzir os custos de desenvolvimento, criando a possibilidade de auxiliar na resolução de possíveis problemas de uma forma simples e direta, por possibilitar o controle desde o controle de versões do código fonte e até disponibilizar bases de e-mails através de listas de mensagens, informações estas de grande importância para a equipe de desenvolvimento e para o treinamento de novos membros que venham a fazer parte do projeto. Ganhos estes conseguidos diretamente a partir de customização e integração das ferramentas de software.

Estima-se que esta nova concepção para desenvolvimento de aplicações em software aliado ao amadurecimento de novas tecnologias poderão sedimentar bases de conhecimento e práticas, fundamentais para alimentar o processo de desenvolvimento de modelos mais eficientes e flexíveis, oferecendo aos clientes soluções mais práticas e funcionais, fazendo com que a tecnologia em questão tenha a possibilidade de agregar valor ao produto final desenvolvido.

\section{Referências}

1. Barbarán, Grabriela Cabel \& Francischini, Paulino Graciano. XIX Encontro Nacional de Engenharia de Produção. Rio de Janeiro, RJ. 1999. Artigo. Gerenciamento de um Projeto de Desenvolvimento de Software. p.04.

2. Maximiniano, Antonio Cesar Amaru. Administração de Projetos, Editora Atlas S.A., 2a. Edição. São Paulo - SP, 2002. p.26

3. Pmbok. Project Management Body of Knowledge. Project Management Institute, 2000.

4. Kanter, R. M. Classe Mundial: Uma agenda para gerenciar os desafios globais em benefício das empresas e das comunidades. Rio de Janeiro: Campus, 1996. p.104

5. Rodrigues, Grabriela Cabel \& Hatakeyama, Kazuo. XXIII Encontro Nacional de Engenharia de Produção. Ouro Preto, MG. 2003. Artigo. A Situação dos Recursos Humanos Aplicados em Manutenção na Região de Curitiba. p.05

6. Pinna, Cristina Coelho de Abreu \& Carvalho, Marly Monteiro. XXII Encontro Nacional de Engenharia de Produção. Curitiba, PR. 2002. Artigo. Gestão de Projetos de Aplicações Web: Uma Abordagem para Minimização de Incertezas na Definição de Escopo. 